\title{
PRESENCIA ESPACIAL Y TEMPORAL DE AVES RAPACES DIURNAS (AVES: ACCIPITRIFORMES, FALCONIFORMES) EN MARISMAS NACIONALES, NAYARIT-SINALOA, MÉXICO
}

\author{
SPATIAL AND TEMPORAL PRESENCE OF DIURNAL RAPTORS \\ (AVES: ACCIPITRIFORMES, FALCONIFORMES) AT MARISMAS NACIONALES, \\ NAYARIT-SINALOA, MEXICO
}

\author{
Roberto CARMONA, ${ }^{1,2, *}$ Luis F. MENDOZA, ${ }^{1}$ David MOLINA, ${ }^{1,2}$ Lidiana ORTEGA, ${ }^{3}$ \\ EMmanuel MIRAMONTES ${ }^{4}$ y Miguel CRUZ ${ }^{4}$

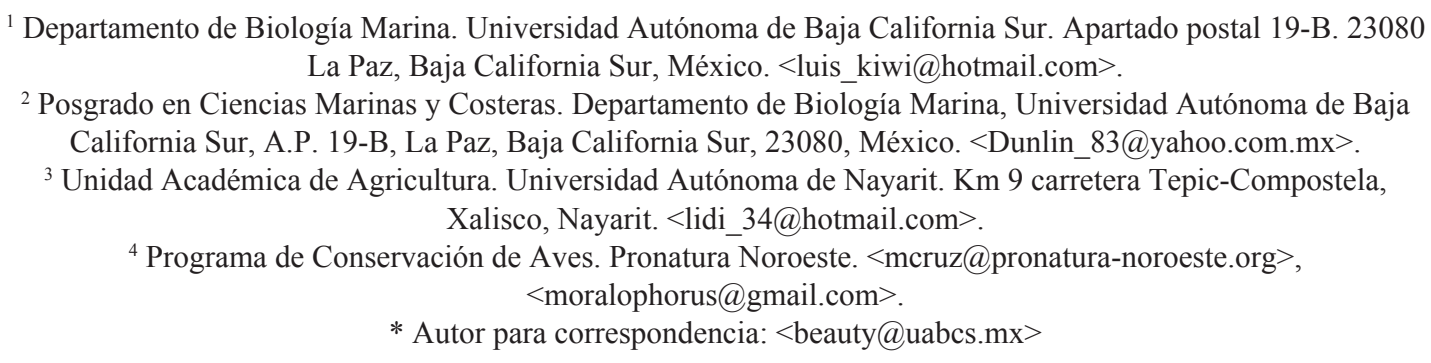

Recibido: 01/03/2016; aceptado: 21/09/2016

Editor responsable: Octavio Rafael Rojas Soto

Carmona, R., Mendoza, L.F., Molina, D., Ortega, L., Miramontes, E. y Cruz, M. (2017) Presencia espacial y temporal de aves rapaces diurnas (Aves: Accipitriformes, Falconiformes) en Marismas Nacionales, Nayarit-Sinaloa, México. Acta Zoológica Mexicana (n.s.), 33(1), 27-38.

RESUMEN. Las aves rapaces diurnas son un grupo esencial para el equilibrio en las comunidades y pueden ser utilizadas como indicadoras de la calidad del hábitat debido a su sensibilidad a la perturbación. Una de las zonas en México con gran importancia biológica y con graves problemas antropogénicos es Marismas Nacionales. Generar información básica de un grupo ornitológico poco estudiado en México y dentro de una zona prioritaria como Marismas Nacionales se hace indispensable para planes de conservación y manejo del área. Por lo que en este estudio se determinó la riqueza espacial y temporal de aves rapaces diurnas durante ocho meses (noviembre de 2010 a junio de 2011) en nueve zonas de Marismas Nacionales. En cada visita se registraron y contabilizaron las especies por sitio y mes. En total se realizaron 945 registros de 22 especies, a las que se añaden cinco más observadas en una visita posterior (enero de 2012) con lo que se tiene una lista de 27 especie. La riqueza en invierno se incrementó con la llegada de cuatro especies migratorias y disminuyó en verano. Las especies más abundantes fueron las de habito necrófago (Cathartes aura, Coragyps atratus) y otra más migratoria (Pandion haliaetus) las cuales conjuntaron en $65 \%$ del total de registros. Las zonas con mayor número de especies fueron Zoquipan y las Garzas-Chahuín con 13 y 11 especies respectivamente. En cuanto a registros totales, Zoquipan y Chumbeño presentaron los más altos con 207 para el primero y 185
Carmona, R., Mendoza, L.F., Molina, D., Ortega, L., Miramontes, E., \& Cruz, M. (2017) Spatial and temporal presence of diurnal raptors (Aves: Accipitriformes, Falconiformes) at Marismas Nacionales, Nayarit-Sinaloa, Mexico. Acta Zoológica Mexicana (n.s.), 33(1), 27-38.

ABSTRACT. Diurnal raptors are an essential group for balance in communities that can be used as habitat quality indicator owing to their sensitivity to disturbance. In Mexico one of the biologically significant areas and that faces serious anthropogenic issues is Marismas Nacionales. Comprehensive management and conservation assessments for the area will require to generate a baseline on poor studied group in Mexico such as raptors. Thus we determined spatial and temporal richness of diurnal raptors through eight months (November 2010 to June 2011) in nine sites of Marismas Nacionales, where we recorded and counted all species per site and month. In that period 945 records of 22 species were made, additionally other five species were observed on a subsequent visit (January 2012) therefore we had 27 species in total. Winter richness increased with the presence of four migratory species and decreased in summer. The most abundant species were the scavengers (Turkey vulture, Black vulture) and one migratory (Osprey); these three species comprised $65 \%$ of total records. We found the highest numbers of species in Zoquipan and Garzas-Chahuín (13 and 11 species respectively). In concern of total records, Zoquipan and Chumbeño presented the highest with 207 for the first and 185 the second one. The results shows a high richness of raptors in Marismas Nacionales due to its geographical position on the limit of two biogeographic regions (transition zone) and secondly by the variety 
el segundo. Los resultados muestran una alta riqueza de aves rapaces en el área de estudio, debido a la posición geográfica, que marca la frontera de dos regiones biogeográficas (zona de transición) y por otro la variedad de ambientes que presenta. La escasa información previa dificulta comparar los eventuales cambios en la composición de las especies, lo que también dificulta magnificar el efecto de la pérdida del hábitat para este grupo.

Palabras claves: Distribución espacial, Reserva de la Biosfera, riqueza, distribución temporal, Marismas Nacionales, Nayarit.

\section{INTRODUCCIÓN}

Las aves de presa o rapaces son un grupo esenciales para mantener el equilibrio en las comunidades, dada su posición tope en la cadena trófica (Mañosa \& Pedrocchi 1997, Rodríguez-Estrella et al. 1998, Vázquez-Pérez et al. 2009). Además, su sensibilidad a la perturbación y a cambios en el ambiente permite que sean utilizadas como especies indicadoras de la calidad del hábitat (Thiollay 1989, Rodríguez-Estrella et al. 1998, Blendinger et al. 2004, Carrete et al. 2009). En México las alteraciones antrópicas a sus hábitats han provocado que la mayoría de las especies de este grupo presenten problemas de conservación, por lo que muchas de ellas ( $80 \%$ del total nacional) tienen algún estatus de protección por parte del gobierno mexicano (DOF 2010a).

La presencia de las aves rapaces diurnas en un determinado ambiente depende de diferentes factores o combinación de ellos, como el estado de conservación del mismo, la disponibilidad de alimento, la presencia de fuentes de perturbación e incluso la época del año, pues tanto los movimientos migratorios como la época de reproducción influyen en la riqueza y abundancia de este grupo (Bustamante \& Seoane 2004, Carrete et al. 2009, Vergara 2010).

Actualmente el cambio de uso de suelo afecta muchos de los ambientes naturales del país, por lo que en algunos casos dichos ambientes se están reduciendo a tasas elevadas (SEMARNAT 2007, López 2012), lo que es aún más notorio en lugares con diferentes actividades económicas (i.e. ganadería, agricultura, acuicultura), como Marismas Nacionales (DOF 2010b, Blanco et al. 2011).

Marismas Nacionales ha sido indicada como una de las zonas de mayor importancia biológica del país (CONANP 2005, Blanco et al. 2011), se localiza en la frontera de los estados de Sinaloa y Nayarit. Está reconocida como Área de Importancia para la Conservación de las Aves (Cervantes-Abrego 2000), sitio clave para la conservación de aves playeras (RHRAP 2015) y la porción naya- of environments in the whole area. The scarce previous data makes difficult to compare any changes in species composition, which also avoid magnify the effect of habitat loss on this group.

Key words: Spatial distribution, biosphere reserve, richness, temporal distribution, Marismas Nacionales, Nayarit.

rita fue designada Reserva de la Biosfera (DOF 2010b). Para la zona correspondiente a Sinaloa se están llevando a cabo las gestiones necesarias para su nombramiento (CONANP 2008).

Para Marismas Nacionales los escasos estudios ornitológicos se han enfocado principalmente a las comunidades de aves acuáticas y la información del componente terrestre está limitada, incluidas las rapaces. Recientemente fueron publicados dos trabajos en los que son mencionadas aves rapaces de la zona, en uno de ellos se informa de la presencia de Gavilanes caracoleros (Rostrhamus sociabilis Vieillot, 1817) y se analiza su distribución espacio-temporal (Carmona et al. 2013); en el otro se indica por primera vez para la zona la presencia invernal de grupos numerosos de Aguililla de Swainson (Buteo swainsoni Bonaparte, 1838) en campos agrícolas de la zona (Mendoza et al. 2016). Sin embargo no hay trabajos que aborden toda la comunidad de aves rapaces en Marismas Nacionales.

Para Nayarit, Escalante (1988) registró 22 especies de aves rapaces diurnas mientras que Howell \& Webb (1995) reportan 32 especies; la plataforma de observación de aves eBrid (consultada en noviembre de 2015) registra para el estado 34 especies de aves rapaces diurnas, pero varias especies son ocasionales (e.g. Aguililla real, Milano de Misisipi). Adicionalmente en 2014 se presentó un listado de las aves de la Sierra de Vallejo, ubicada al sur de Nayarit, en él se incluyen 24 especies de aves rapaces diurnas (Figueroa-Esquivel \& Puebla-Olivares 2014). Para Sinaloa existen registros de 28 especies de aves rapaces (Howell \& Webb 1995). Tanto por la importancia del grupo taxonómico, como por la de Marismas Nacionales no es justificable la falta de información existente, sobre todo al considerar la serie de actividades económicas que se realizan en Marismas Nacionales (e.g. ganadería, agricultura, acuicultura), las cuales pueden ocasionar alteraciones en el entorno, sin que exista una línea base que permita comparar cambios en la comunidad de las aves rapaces de la zona. 
Por lo anterior el objetivo de este trabajo fue describir la riqueza espacial y temporal de las aves rapaces diurnas en Marismas Nacionales a lo largo de ocho meses.

\section{MATERIALES Y MÉTODOS}

Área de Estudio. Marismas Nacionales es un extenso sistema de humedales que se localiza entre el noroeste de Nayarit y el sur de Sinaloa, en la costa del Pacífico Mexicano (Fig. 1). Tiene una superficie de 300000 ha, de las cuales 133854 ha están incluidas dentro de la Reserva de la Biosfera homónima en Nayarit (DOF 2010b). El uso de suelo en la región es predominantemente agropecuario. La zona es una red de lagunas costeras salobres, manglares, planicies lodosas, cañadas y marismas (Saunders \& Saunders 1981, Carmona et al. 2013). Marismas Nacionales incluye los bosques de manglar más extenso del Pacífico oriental mexicano (Berlanga-Robles \& RuizLuna 2006).

La zona presenta un clima cálido subhúmedo con lluvias en verano. Las lluvias son abundantes y rara vez inferiores a $1000 \mathrm{~mm}$ anuales, se concentran de junio a octubre, época en las que en promedio se precipitan 964 mm (92\%; CONAGUA 2004). La vegetación predominante es de bosque de manglar, vegetación halófita rastrera (Salicornia spp. Linnaeus, 1753 y Batis maritima Linnaeus, 1759), selva baja perennifolia, palma de aceite (Orbignya spp. Martius, 1837), selva baja caducifolia, pastizales y potreros, cultivos de frutales y hortalizas. La vegetación acuática está representada por lirio acuático (Eichhornia crassipes Solms, 1883), sauces (Salix spp. Linnaeus, 1753) y tule (Typha latifolia Linnaeus, 1753), entre otras (Carmona et al. 2013).

Se eligieron nueve áreas, tratando de incluir todos los ambientes presentes (como bosque de manglar, lagunas, planicies lodosas y marismas; Fig. 1):

1) Zoquipan se ubica al sur de Marismas Nacionales; incluye un canal principal y cuatro cuerpos de agua, dos de ellos estacionales. Presenta un gradiente de salinidades que va de la boca (altas salinidades) hacia el interior. La vegetación de este sitio incluye manglares (Rhizophora mangle Linnaeus, 1753) que cubren el perímetro de los canales y la mayor parte de las lagunas, donde también existen zonas de tule (T. latifolia). El lirio acuático cubre una extensa superficie del cuerpo de agua (INEGI 2000).
2) La Batanga es una laguna salobre estacional que se comunica con otros cuerpos de agua por medio de canales de poca profundidad. La vegetación predominante es el manglar y plantas halófitas (Salicornia spp. y B. maritima).

3) La zona de Mexcaltitan comprende una extensa laguna continental (902 ha), que es el cuerpo principal y otras dos más pequeñas localizadas en sus adyacencias. La profundidad es baja (menor a $1 \mathrm{~m}$ ) en la mayor parte de su extensión. La vegetación predominante es el manglar; el lirio cubre amplias áreas del espejo de agua. La salinidad es baja en la época de lluvias y se incrementa en el estiaje.

4) Las Cañadas es uno de los complejos estuarinos más grande en la costa del Pacífico mexicano (Kovacs et al. 2008). Este sistema cuenta con numerosas lagunas salobres de poca profundidad, formadas por la presencia de aproximadamente 150 barras de arena semiparalelas, en las que existe vegetación de manglar (Avicennia germinans Linnaeus, 1764 y Laguncularia racemosa L. Gaetner, 1805). Las barras son utilizadas como áreas de cultivos y pastoreo.

5) La laguna El Chumbeño es un humedal salobre localizado a $32 \mathrm{~km}$ de la costa y que se interconecta a través de una red de esteros y canales al sistema lagunar Teacapán-Agua Brava (40 000 ha). A orillas de la laguna se encuentran dos poblados: Pescadero y Francisco Villa, que en conjunto cuentan con 1700 habitantes dedicados a la pesca de camarón principalmente (Güemez-Álvarez 2011).

6) Las lagunas Las Garzas-Chahuin Chihua se ubican en la porción norte de Marismas Nacionales. Con un perímetro interno de aproximadamente $15 \mathrm{~km} \mathrm{y}$ a una distancia de $10 \mathrm{~km}$ de la línea de costa (Carmona et al. 2011). Presentan llanuras arenosas y lodosas, islas artificiales creadas por el dragado de canales, una salinera usada ocasionalmente, vegetación halófita (Salicornia spp. y B. maritima) y manglar en baja densidad.

7) La zona de Zoyata está formada por tres lagunas continuas de escasa profundidad (menor a $1 \mathrm{~m}$ ) en la mayor parte de su extensión, presenta canales que son utilizados para la navegación de embarcaciones menores donde la profundidad es de $2 \mathrm{~m}$. La vegetación está conformada por manglares y tulares. Durante la temporada de lluvias estas lagunas presentan salinidades bajas, pero conforme se drenan la salinidad aumenta. 


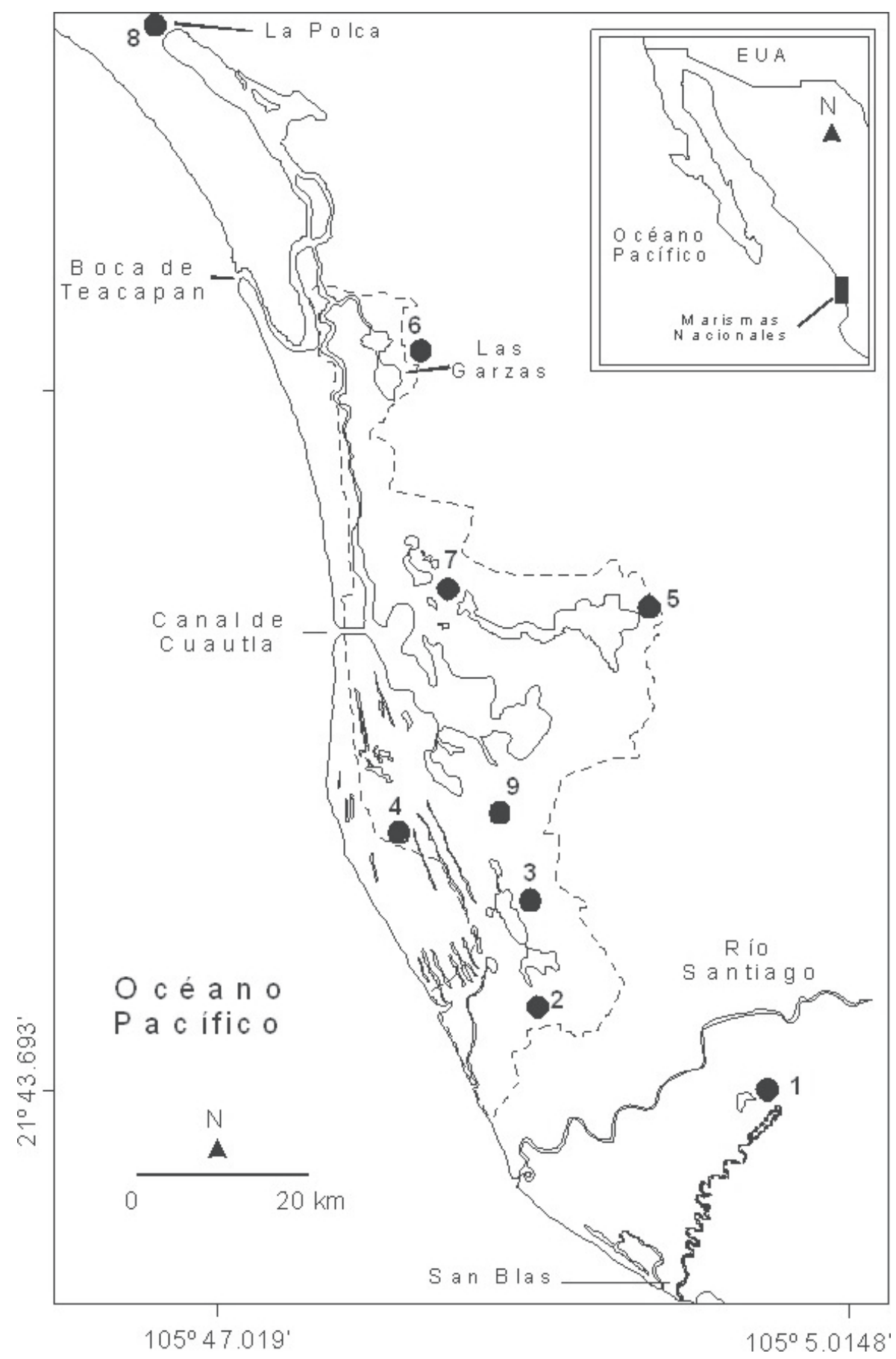

Figura 1. Área de estudio. Se indican los sitios de observación visitados (1) Zoquipan, (2) La Batanga, (3) Mexcaltitán, (4) Las Cañadas, (5) Chumbeño, (6) Las Garzas-Chahuín, (7) Zoyata, (8) La Polca y (9) Los Espejos. La línea punteada representa el área designada como Reserva de la Biosfera.

8) La Polca forma parte de un sistema lagunar al que se suman La Estacada y Puerta de México, ubicada en Sinaloa. La laguna es somera de forma rectan- gular y con una superficie cercana a las 1200 ha, se ubica a $7 \mathrm{~km}$ de la costa y tiene comunicación al mar por la Boca de Teacapán. Hacia su porción 
sureste se localizan mangles ( $R$. mangle) dispersos. En la periferia de la laguna existen áreas de cultivo y palmares.

9) El Embarcadero los Espejos presenta una laguna estacional y una permanente. La vegetación predominante es el mangle (A. germinans y $R$. mangle) y la marisma (Salicornia spp.). Existen canales artificiales que facilitan el tránsito de embarcaciones menores.

Toma de datos y Análisis. El proyecto bajo el cual se realizó la toma de datos estuvo enfocado a las aves acuáticas, sin embargo, a lo largo de los recorridos se identificaron y contabilizaron todas las aves rapaces observadas. Dado que no se aplicó una metodología propia para este grupo (e.g. Fuller \& Mosher 1987) existe una alta probabilidad de que los datos cuantitativos generados estén subestimados, por lo que a este respecto los análisis realizados fueron limitados, pese a lo anterior, los datos cualitativos (riquezas por tiempo y zona) son robustos y sobre estos se centran la mayor parte de los análisis y discusión.

Los nueve sitios seleccionados se visitaron mensualmente entre noviembre de 2010 y junio de 2011. En cada visita se formaron dos equipos de trabajo, uno de los cuales recorrió el sistema norte de Marismas Nacionales (La Polca) y el otro el sur (Laguna de Zoquipan), para encontrarse en Laguna Las Garzas-Chahuín (Fig. 1). Los recorridos fueron de tres tipos: lancha, automóvil y por caminatas y su uso dependió de la naturaleza del terreno. Las observaciones empezaron a las 07:00 h y terminaron a las 17:00 h, cada visita tuvo una duración de 5 días, por lo que los datos presentados representan 80 días de trabajo de campo. Las especies se identificaron utilizando diferentes guías de campo (Howell \& Webb 1995, van Perlo 2006, Dunn \& Alderfer 2011). Las observaciones se realizaron con binoculares (8 y 10x) $\mathrm{y}$ telescopio (15-60x), además se tomaron fotografías de la mayoría de las especies. Adicionalmente se realizó una visita en enero de 2012, los registros nuevos se incluyeron únicamente en el listado, sin utilizarse en los análisis.

Se presenta la lista de aves rapaces diurnas, incluido su estatus (residente, invernal, transitorio e indeterminado; Howell \& Webb 1995, Iliff \& Lovitch 2007) y su estatus de conservación (amenazado y protección especial; DOF 2010a). Los nombres comunes de las aves se tomaron de Escalante et al. (2014). La nomenclatura científica y el orden sistemático son los establecidos por la AOU (1998) y suplementos posteriores.
Los análisis sobre presencia-ausencia incluyeron dos pruebas de independencia $X^{2}$ con dos criterios de clasificación (Siegel \& Castellan 1988), en las que se determinó si la riqueza observada por mes y por zona (por separado), puede considerase independiente de estos criterios $(\alpha=0.05$, en ambos casos). Además se realizaron dendrogramas de similitud, empleando el coeficiente de Sorensen (presencia/ausencia) para los ocho meses y las nueve zonas por separado (Krebs 1989, Clarke \& Warwick 2001), utilizando el análisis de unión media no ponderada (Crisci \& López 1983). En ambos casos se consideró el 70\% de similitud como el punto de corte para integrar grupos homogéneos, porcentaje superior al 50\% indicado en algunos estudios (e.g. Fay et al. 1977).

Respecto a los datos cuantitativos únicamente se estimó para cada especie el índice de importancia relativa (Gatto et al. 2005), el cual se define como $I I R=100\left(N_{i} /\right.$ $\left.N_{t}\right)\left(M_{i}+E_{i}\right) /\left(M_{t}+M_{t}\right)$. Donde $N_{i}$ es la suma de las abundancias de las especies $i$ en los diferentes meses, $N_{t}$ es la suma total de las abundancias, $M_{i}$ es el número de meses donde la especie $i$ estuvo presente, $E_{i}$ es el número de sitios donde la especie $i$ estuvo presente, $M_{t}$ y $E_{t}$ son el número total de meses y sitios de muestreo, respectivamente (Gatto et al. 2005). Este índice pondera las frecuencias de ocurrencia temporal y espacial de cada especie con su abundancia proporcional. La suma total de los índices para un grupo determinado de especies es 100 , por lo que permite una comparación interespecífica sencilla.

\section{RESULTADOS}

Durante ocho meses se registraron 22 especies de rapaces diurnas, de las familias Cathartidae (2 especies), Pandionidae (1), Accipitridae (14) y Falconidae (5; Cuadro 1). Para todas las especies se realizaron 945 registros (Cuadro 1), que al considerar 80 días de trabajo de campo, implicó un promedio de 11.8 aves rapaces/día. De acuerdo a la Norma Oficial Mexicana, nueve especies se encuentran listadas en la categoría de protección especial y una más como amenazada (DOF 2010a; Cuadro 1). 14 de las 22 especies fueron residentes (63\%), siete migratorias (32\%) y una con estatus indeterminado (5\%; Cuadro 1). Esta última especie corresponde al gavilán caracolero $(R$. sociabilis) que presentó 109 (11.5\%) registros (Cuadro 1).

A las 22 especies anteriores se adicionan cinco más que fueron registradas en enero de 2012: el Gavilán pico gancho (Chondrohierax uncinatus Temminck, 1822; 12 enero, protección especial), Gavilán de Cooper (Accipi- 
Cuadro 1. Abundancia mensual de aves rapaces diurnas en Marismas Nacionales. Se indican sus registros totales, su frecuencia de ocurrencia mensual, el número de zonas en las que fueron observadas, su índice de importancia relativa (IIR) y su estatus $(\mathrm{R}=$ residente, $\mathrm{M}=$ migratorio, $\mathrm{T}=$ transeúnte y $\mathrm{ID}=$ indeterminado). Para especies en la Norma Oficial Mexicana $(\mathrm{NOM}-059)$ se indica su categoría al final del nombre $(\mathrm{Pr}=$ protección especial y $\mathrm{A}=$ amenazada).

\begin{tabular}{|c|c|c|c|c|c|c|c|c|c|c|c|c|c|}
\hline \multicolumn{14}{|c|}{ MESES } \\
\hline Especies & Nov & Dic & Ene & Feb & Mar & Abr & May & Jun & Total & Frecuencia & Zonas & IIR & Estatus \\
\hline Coragyps atratus & 123 & 9 & 0 & 2 & 7 & 2 & 7 & 4 & 154 & 7 & 8 & 16.64 & $\mathrm{R}$ \\
\hline Cathartes aura & 54 & 53 & 95 & 35 & 40 & 15 & 5 & 0 & 297 & 7 & 9 & 34.23 & $\mathrm{R}$ \\
\hline Pandion haliaetus & 16 & 14 & 37 & 44 & 35 & 8 & 5 & 3 & 162 & 8 & 9 & 19.84 & $\mathrm{M}$ \\
\hline Elanus leucurus & 0 & 0 & 0 & 2 & 0 & 0 & 0 & 0 & 2 & 1 & 1 & 0.03 & $\mathrm{R}$ \\
\hline Rostrhamus sociabilis $^{\mathrm{Pr}}$ & 23 & 7 & 22 & 17 & 8 & 16 & 13 & 3 & 109 & 8 & 3 & 8.64 & ID \\
\hline Circus cyaneus & 0 & 1 & 0 & 1 & 1 & 0 & 0 & 0 & 3 & 3 & 3 & 0.13 & M \\
\hline Geranospiza caerulescens $^{\mathrm{A}}$ & 0 & 1 & 1 & 1 & 0 & 0 & 0 & 0 & 3 & 3 & 4 & 0.13 & $\mathrm{R}$ \\
\hline Buteogallus anthracinus $^{\mathrm{Pr}}$ & 13 & 14 & 15 & 25 & 5 & 4 & 1 & 4 & 81 & 8 & 8 & 9.34 & $\mathrm{R}$ \\
\hline Buteogallus urubitinga $a^{\mathrm{Pr}}$ & 1 & 0 & 0 & 0 & 0 & 0 & 0 & 0 & 1 & 1 & 1 & 0.01 & $\mathrm{R}$ \\
\hline Parabuteo unicinctus $^{\mathrm{Pr}}$ & 0 & 0 & 2 & 0 & 1 & 1 & 3 & 0 & 7 & 4 & 2 & 0.30 & $\mathrm{R}$ \\
\hline Buteo plagiatus & 0 & 0 & 4 & 17 & 0 & 2 & 1 & 0 & 24 & 4 & 7 & 1.90 & $\mathrm{R}$ \\
\hline Buteo brachyurus & 0 & 5 & 4 & 0 & 0 & 0 & 0 & 1 & 10 & 3 & 2 & 0.36 & $\mathrm{R}$ \\
\hline Buteo swainsoni $i^{\mathrm{Pr}}$ & 0 & 0 & 3 & 0 & 0 & 0 & 0 & 0 & 3 & 1 & 3 & 0.09 & $\mathrm{~T}$ \\
\hline Buteo albicaudatus ${ }^{\mathrm{Pr}}$ & 1 & 0 & 0 & 0 & 1 & 0 & 0 & 0 & 2 & 2 & 2 & 0.06 & $\mathrm{R}$ \\
\hline Buteo albonotatus $^{\mathrm{Pr}}$ & 0 & 0 & 0 & 0 & 0 & 1 & 0 & 0 & 1 & 1 & 1 & 0.01 & M \\
\hline Buteo jamaicensis & 1 & 1 & 0 & 0 & 0 & 0 & 0 & 0 & 2 & 2 & 1 & 0.04 & $\mathrm{R}$ \\
\hline Micrastur semitorquatus $^{\mathrm{Pr}}$ & 0 & 0 & 0 & 0 & 0 & 1 & 0 & 0 & 1 & 1 & 1 & 0.01 & $\mathrm{R}$ \\
\hline Caracara cheriway & 18 & 2 & 7 & 6 & 2 & 8 & 6 & 1 & 50 & 8 & 9 & 6.12 & $\mathrm{R}$ \\
\hline Herpetotheres cachinnans & 0 & 1 & 0 & 0 & 0 & 0 & 0 & 0 & 1 & 1 & 1 & 0.01 & $\mathrm{R}$ \\
\hline Falco sparverius & 2 & 0 & 3 & 6 & 0 & 0 & 0 & 0 & 11 & 3 & 5 & 0.63 & M \\
\hline Falco columbarius & 1 & 0 & 0 & 0 & 0 & 0 & 0 & 0 & 1 & 1 & 1 & 0.01 & $\mathrm{M}$ \\
\hline Falco peregrinus $^{\mathrm{Pr}}$ & 2 & 1 & 6 & 7 & 4 & 0 & 0 & 0 & 20 & 5 & 5 & 1.44 & M \\
\hline Riqueza de especies & 12 & 12 & 12 & 12 & 10 & 10 & 8 & 6 & 22 & & & & \\
\hline Abundancia & 255 & 109 & 199 & 163 & 104 & 58 & 41 & 16 & 945 & & & & \\
\hline & 12 & 4 & 3 & 1 & 0 & 2 & 0 & 0 & & & & & \\
\hline
\end{tabular}

ter cooperii Bonaparte, 1828; 12 enero, protección especial), Halcón enano (Falco rufigularis Daudin, 1800; 12 enero), Gavilán pecho rufo (A. striatus Vieillot, 1808; 14 enero, protección especial) y el Aguililla ala ancha ( $B$. platypterus Vieillot, 1823; 14 de enero, protección especial).

La riqueza específica fue dependiente del mes de observación $\left(X_{7}{ }_{7}=25.2, \mathrm{p}<0.01\right)$. Dicha variable se mantuvo estable y alta entre noviembre y febrero (12 especies), presentó valores medios en marzo y abril (10) y bajos en mayo y junio ( 8 y 6 , respectivamente; Cuadro 1 ). El dendrograma de riqueza temporal agrupó los meses de invierno (diciembre a febrero), los de primavera (marzo a mayo) y con menor similitud los meses extremos, noviembre (otoño) y junio (verano; Fig. 2).

De forma similar a los resultados temporales, la riqueza específica tampoco pudo ser considerada independiente de la zona de observación $\left(X_{8}^{2}=34.2, p<0.01\right)$. Espacialmente la riqueza fluctuó entre siete y 13 especies (Cuadro 2). La zona con mayor riqueza fue Zoquipan con 13 especies y las de menor riqueza fueron Zoyata y Los Espejos con siete especies cada una (Cuadro 2). Espacialmente el dendrograma (Fig. 3) agrupó tres zonas ubicadas en la parte central de Marismas Nacionales (La Batanga, Mexcaltitan y Los Espejos), cuatro zonas norteñas (Cañadas, Zoyata, Chumbeño y La Polca) y dos zonas que no se 


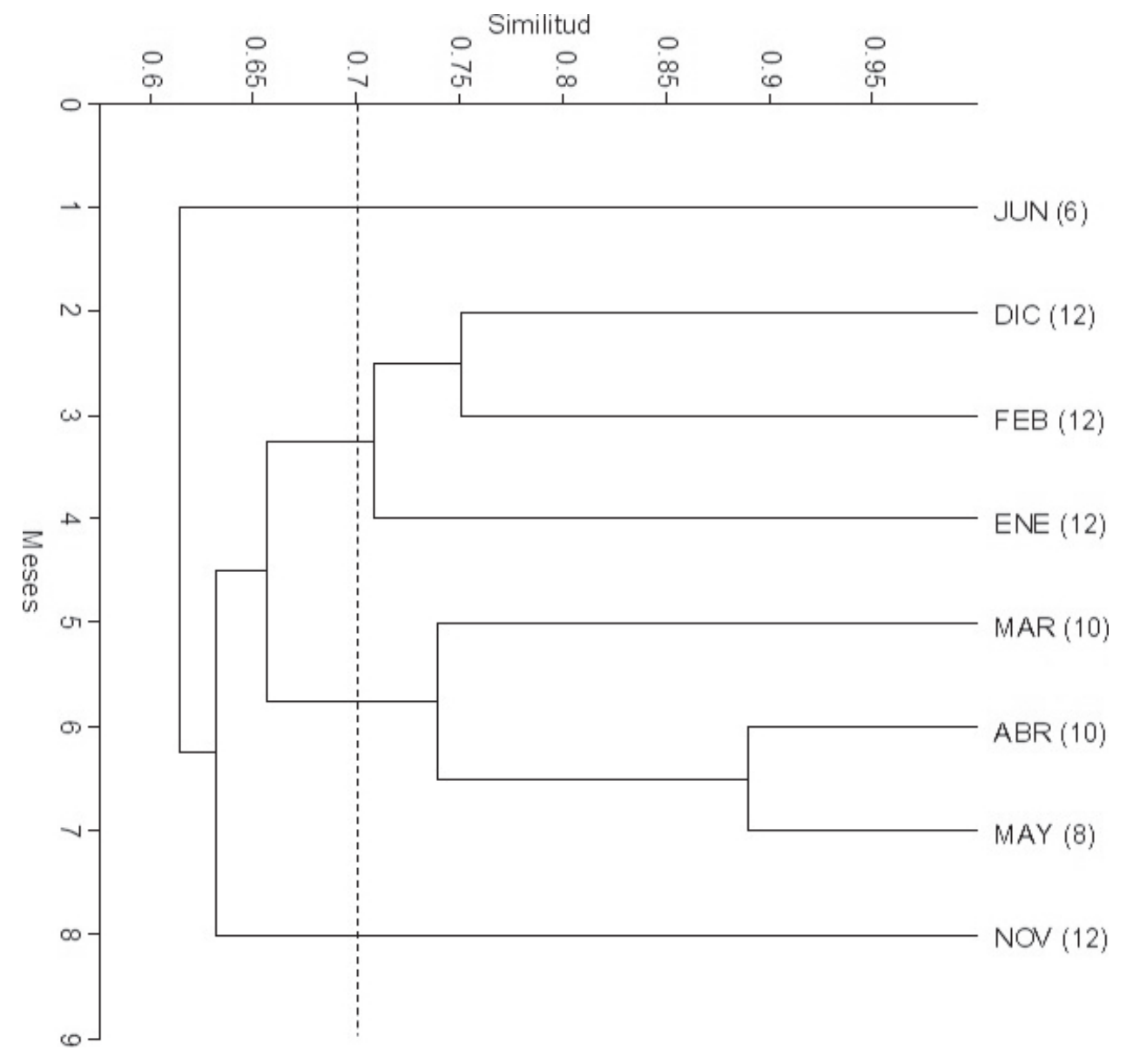

Figura 2. Dendrograma de similitud temporal. Al final de la abreviatura de los meses se indica entre paréntesis el número de especies vistas.

integraron a estos grupos, la más sureña (Zoquipan) y otra ubicada al norte del sistema (Las Garzas).

Respecto al número de aves por salida se observan dos períodos, el de octubre a marzo con más de 100 registros por mes y el de abril a junio con una disminución notoria en éstos (Cuadro 1). Seis especies fueron las más comúnmente observadas y con los valores mayores de índice de importancia relativa (IIR); estas fueron en orden decreciente, el Zopilote aura (Cathartes aura Linnaeus, 1578; 297 registros y 34.23 IIR), el Gavilán pescador (Pandion haliaetus Linnaeus, 1578; 162 y 19.82), el Zopilote común (Coragyps atratus Bechstein, 1793; 154 y 16.64), el Aguililla negra menor (Buteogallus anthracinus Deppe, $1830 ; 81$ y 9.34), el Gavilán caracolero ( $R$. sociabilis; 109 y 8.64) y el Caracara quebrantahuesos (Caracara cheriway Jacquin, 1784; 50 y 6.12). Estas especies en conjunto acumularon cerca del $95 \%$ del índice de impor- tancia relativa y poco más del $90 \%$ de los registros totales (Cuadro 1).

Con respecto a las seis especies más comunes, cuatro fueron residentes y dentro de estas, tres presentan hábitos necrófagos; una especie fue invernante y una más tiene un estatus indeterminado (Cuadro 1). La abundancia de aves rapaces varió entre las zonas, puesto que en tres de ellas se registró el $60 \%$ de los registros: Zoquipan, Mexcaltitan y Chumbeño (Cuadro 2). Este último sitio presentó 185 registros y una dominancia de especies necrófagas (91\%; Cuadro 2).

Tres especies mostraron clara preferencia por alguna (s) zona (s). El 78\% de los registro de la Aguililla negra menor se llevaron a cabo en Zoquipan, Mexcaltitan y Las Cañadas; el 73\% de las observaciones del Gavilán pescador se realizaron en Zoquipan, Mexcaltitan y Laguna Las Garzas-Chahuín; por último el $96 \%$ de los registros del 
Cuadro 2. Número de registros totales por zona para las aves rapaces diurnas observadas en Marismas Nacionales. Se indica el total de registros por especie (Total). Al final de la tabla se indica la Abundancia y la riqueza totales por zona.

\begin{tabular}{lcccccccccc}
\hline \multicolumn{1}{c}{ Especies } & Zoq & Bat & Mex & Cañ & Chum & Gar & Zoy & Pol & Esp & Total \\
\hline Coragyps atratus & 13 & 5 & 4 & 1 & 118 & 11 & 1 & 1 & 0 & 154 \\
Cathartes aura & 28 & 23 & 46 & 15 & 36 & 42 & 12 & 94 & 1 & 297 \\
Pandion haliaetus & 45 & 8 & 43 & 6 & 11 & 31 & 12 & 5 & 1 & 162 \\
Elanus leucurus & 0 & 0 & 0 & 0 & 0 & 2 & 0 & 0 & 0 & 2 \\
Rostrhamus sociabilis & 72 & 4 & 33 & 0 & 0 & 0 & 0 & 0 & 0 & 109 \\
Circus cyaneus & 0 & 1 & 0 & 0 & 0 & 1 & 0 & 1 & 0 & 3 \\
Geranospiza caerulescens & 1 & 1 & 1 & 0 & 0 & 0 & 0 & 0 & 0 & 3 \\
Buteogallus anthracinus & 27 & 7 & 16 & 20 & 1 & 7 & 2 & 0 & 1 & 81 \\
Buteogallus urubitinga & 0 & 0 & 0 & 0 & 0 & 1 & 0 & 0 & 0 & 1 \\
Parabuteo unicinctus & 0 & 0 & 0 & 6 & 0 & 1 & 0 & 0 & 0 & 7 \\
Buteo plagiatus & 3 & 4 & 1 & 12 & 0 & 0 & 2 & 1 & 1 & 24 \\
Buteo brachyurus & 9 & 0 & 0 & 0 & 1 & 0 & 0 & 0 & 0 & 10 \\
Buteo swainsoni & 0 & 0 & 1 & 0 & 0 & 1 & 0 & 0 & 1 & 3 \\
Buteo albicaudatus & 1 & 0 & 0 & 0 & 0 & 0 & 0 & 1 & 0 & 2 \\
Buteo albonotatus & 0 & 0 & 0 & 0 & 1 & 0 & 0 & 0 & 0 & 1 \\
Buteo jamaicensis & 2 & 0 & 0 & 0 & 0 & 0 & 0 & 0 & 0 & 2 \\
Micrastur semitorquatus & 1 & 0 & 0 & 0 & 0 & 0 & 0 & 0 & 0 & 1 \\
Caracara cheriway & 4 & 3 & 1 & 10 & 15 & 3 & 2 & 8 & 4 & 50 \\
Herpetotheres cachinnans & 1 & 0 & 0 & 0 & 0 & 0 & 0 & 0 & 0 & 1 \\
Falco sparverius & 0 & 1 & 0 & 6 & 1 & 0 & 0 & 2 & 1 & 11 \\
Falco columbarius & 0 & 0 & 0 & 1 & 0 & 0 & 0 & 0 & 0 & 1 \\
Falco peregrinus & 0 & 0 & 0 & 3 & 1 & 9 & 1 & 6 & 0 & 20 \\
Abundancia total por zona & 207 & 57 & 146 & 80 & 185 & 109 & 32 & 119 & 10 & 945 \\
Riqueza total por zona & 13 & 10 & 9 & 10 & 9 & 11 & 7 & 9 & 7 & \\
\hline
\end{tabular}

Gavilán caracolero fueron llevados a cabo en Zoquipan y Mexcaltitan (Cuadro 2).

\section{DISCUSIÓN}

La riqueza registrada fue similar a lo reportado para la selva tropical de Chiapas (Ramírez-Albores 2010; 25 especies) y para la selva veracruzana (Ortiz-Pulido et al. 1995; 25 especies) y superior a los valores registrados para la selva del Ocote, Chiapas (Vázquez- Pérez et al. 2009; 16 especies), para la selva baja de Oaxaca (Vázquez et al. 2009; 11 especies) y para la sierra de Huautla, Morelos (Ramírez-Albores \& Ramírez-Cedillo 2002; 16 especies). Al comparar con los registro de Sierra de Vallejo al sur de Nayarit (Figueroa-Esquivel \& Puebla-Olivares 2014), la riqueza total fue semejante (27 vs 24 especies), pero en el trabajo presente se observaron especies que prefieren zonas abiertas y cuerpos de agua (como P. haliaetus y $R$. sociabilis); en contraste en Sierra de Vallejo se vieron especies con afinidad por bosques, como Spizaetus tyrannus Wied, 1820.

El número de especies visto en este trabajo es elevado, lo que de acuerdo a la literatura, se relaciona con dos factores; por una parte la variedad de ambientes que ofrece Marismas Nacionales y que incluyen: selva baja, manglares, planicies lodosas y campos de cultivo entre otros, esta heterogeneidad permite una mayor presencia de especies de aves en general, incluidas las rapaces (Anderson 2001). Por otra parte, la posición geográfica de 


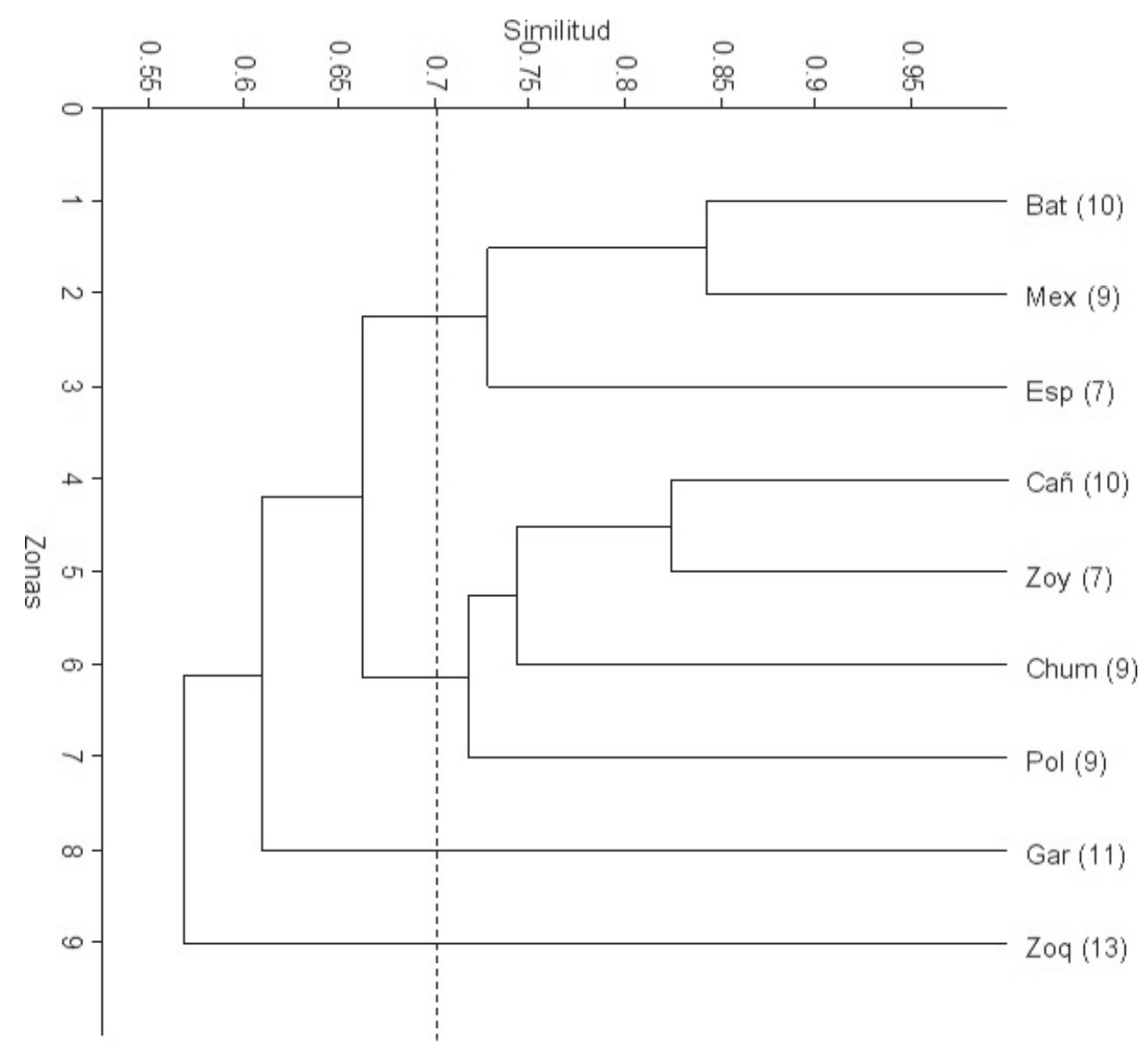

Figura 3. Dendrograma de similitud espacial. Al final de la abreviatura de las zonas se indica entre paréntesis el número de especies vistas.

Nayarit, ya que representa una zona de transición donde convergen especies rapaces del Neotrópico y del Neártico (Ferguson-Lees \& Christie 2001).

Los registros del gavilán caracolero ( $R$. sociabilis) confirman la presencia de la especie en Marismas Nacionales. Se trata de una especie tropical que basa su alimentación en caracoles dulceacuícolas (Pomacea spp. Perry, 1810), por lo que su presencia está estrechamente relacionada con la de este recurso (Sykes et al. 1995, Iliff \& Lovitch 2007). En México, su distribución hasta inicios de los años noventa del siglo pasado, incluía desde la península de Yucatán hasta el centro de Veracruz, con una pequeña conexión a través del Istmo de Tehuantepec a la vertiente del Pacífico (Howell \& Webb 1995). Sin embargo, recientemente se ha notado la presencia de esta especie en diferentes estados del oeste del país, se incluyen Colima, Jalisco y Nayarit (Howell 2004, Palomera-García et al. 2006, Iliff \& Lovitch 2007, Hernández-Vázquez et al. 2013, Carmona et al. 2013), de hecho ya han sido registrados nidos de esta especie en Manzanillo, Colima (Palomera-García et al. 2006). Actualmente es una rapaz común en Marismas Nacionales (Carmona et al. 2013), aunque no se tienen aún evidencias de su reproducción.

Respecto al Aguililla ala ancha, es un especie que se reproduce desde la parte centro-sur de Canadá, a lo largo de los estados del este de Estados Unidos, al sur hasta Texas (Goodrich et al. 1996); en invierno, su área de presencia norteña inicia al sur de Nayarit (Howell \& Webb 1995), aunque otros autores la marcan hasta Colima (Goodrich et al. 1996), independientemente de la exactitud geográfica, la parte norte de Nayarit no está incluida en dicho intervalo; por lo que este es el primer registro para Marismas Nacionales.

Las tres especies ampliamente distribuidas en tiempo y espacio correspondieron a aves necrófagas (Cathartes aura y Coragyps atratus) y al Gavilán pescador (Pandion haliaetus) y son las que presentaron los índices de importancia relativa más altos. Estas especies comparten 
un grado alto de tolerancia a actividades humanas, un patrón semejante se describió en la selva el Ocote, Chiapas (Vázquez-Pérez et al. 2009) y para sistemas subtropicales guatemaltecos (Vannini 1989).

Respecto a la riqueza temporal entre noviembre y febrero se presentaron números altos de especies (12), con las especies migratorias bien representadas (como $B$. swainsoni, F. sparverius Linnaeus, 1758 y $F$. peregrinus Tunstall, 1771), para las dos últimas especies se ha indicado que usan la zona para invernar (Escalante 1988, Howell \& Webb 1995, Smallwood \& Bird 2002). Otra especie migratoria notoria es el Aguililla de Swainson (B. swainsoni), ya que la literatura indica al noroeste de México, Nayarit incluido (e.g. Howell \& Webb 1995) como un área de paso; sin embargo, recientemente se ha registrado su presencia en invierno al menos en el sur de la Península de Baja California (Rodríguez-Estrella et al. 2011) y para Marismas Nacionales (Mendoza et al. 2016), en ambos casos utiliza para su alimentación campos agrícolas.

Otro grupo temporal lo formaron los meses de primavera (marzo a mayo) con un número menor de especies y con una representación claramente a la baja de las migratorias, lo que concuerda con sus fechas de partida al norte (Ferguson-Lees \& Christie 2001). En los extremos de estos períodos se ubicó noviembre, como reflejo de movimientos otoñales y junio, mes ya de verano en el que la riqueza fue muy baja. En mayo y junio sólo estuvo presente una especie migratoria, el Gavilán pescador, para la que se ha documentado el veraneo de aves juveniles y subadultas en área típicas de invernada (Henny \& Van Velze 1972).

Espacialmente las nueve zonas estudiadas cubrieron prácticamente todos los ambientes naturales existentes en Marismas Nacionales. En Zoquipan los valores altos de riqueza y abundancia se relacionan con la estructura del paisaje (Hunt 1987, Vázquez-Pérez et al. 2009), dado que en el área existen bosque de manglar, profusos palmares y marismas, entre otros, los cuales además se encuentran en general poco perturbados (Berlanga-Robles \& Ruiz-Luna 2006); además Zoquipan fue el único sitio que presentó registros de especies asociadas a selva como el Halcón selvático de collar (Micrastur semitorquatus Vieillot, 1817) y el Halcón guaco (Herpetotheres cachinnans Linnaeus, 1758). Por último en esta zona también se realizaron la mayor parte de los registros de Gavilán caracolero, de hecho actualmente es considerada una especie común al sur de Marismas Nacionales (Iliff \& Lovitch 2007, Carmona et al. 2013).
En las Garzas-Chahuin, el segundo sitio en número de especies, fue notoria la presencia de aves con preferencia por ambientes abiertos, como el Aguililla de Swainson, el Gavilán rastrero (Circus cyaneus Linnaeus, 1766), el Milano cola blanca (Elanus leucurus Vieillot, 1818) y el Halcón peregrino ( $F$. peregrinus). Los campos agrícolas y los extensos salitrales que bordean la laguna favorecieron la presencia de estas especies, similar a lo registrado para otros sitios, donde coinciden actividades agrícolas y áreas naturales abiertas (Collopy \& Bildstein 1987, HernándezVázquez et al. 2000, Williams et al. 2000, Pandolfino et al. 2011).

Se ha indicado que las especies carroñeras (e.g. zopilote, caracara) son muy tolerantes a la perturbación e incluso prefieren áreas cercanas a poblados (RodríguezEstrella et al. 1998, Sánchez-Zapata \& Calvo 1999, Zurita \& Bellocq 2007, Vázquez-Pérez et al. 2009), lo que fue coincidente con las observaciones presentes, puesto que estas especies fueron particularmente comunes y abundantes en los sitios con ambientes modificados como Chumbeño y la Polca.

La escasa información existente dificulta comparar los resultados presentes para poder evaluar cambios eventuales en la composición de las especies de aves rapaces en Marismas Nacionales. Sin embargo, este trabajo registró una de las mayores riquezas indicadas para el país, por lo que es prioritario establecer planes de monitoreo que permitan detectar modificaciones de la estructura comunitaria ante la pérdida o modificación del hábitat en la región.

AGRADECIMIENTOS. A Pronatura Noroeste oficina Tepic particularmente a Mauricio Cortés y César Rodríguez por su apoyo en el trabajo de campo. Este trabajo fue parcialmente apoyado por el Proyecto "Planificación de mecanismo para la conservación legal y restauración de las poblaciones de aves acuáticas migratorias invernantes en Marismas Nacionales, Nayarit, México", auspiciado por North American Wetland Conservation Act, agradecemos particularmente el apoyo de Ellen Murphy. También se recibió apoyo de Kennecott Utah Copper Corporation, and the Rio Tinto-Bird Life Program, por lo que se agradece a Jonathan Stacey e Ítala Yepes, al equipo del Secretariado de las Américas de BirdLife International y a los miembros de Linking Communities. Al personal de la Reserva de la Biosfera Marismas Nacionales, Nayarit, por la ayuda brindada a lo largo del trabajo de campo, en particular a Víctor Hugo Vázquez Morán (director) y Daniela Valera Aguilar (responsable del programa de monitoreo).

\section{LITERATURA CITADA}

American Ornithologists' Union (AOU) 1998. Check-list of North American Birds. Allen Press, Lawrence, Kansas, 829 pp. 
Anderson, D. L. 2001. Landscape heterogeneity and diurnal raptor diversity in Honduras: the role of indigenous Shifting cultivation. Biotropica, 33, 511-519.

Berlanga-Robles, C. A. \& Ruiz-Luna, A. 2006. Evaluación de cambios en el paisaje y sus efectos sobre los humedales costeros del sistema estuarino de San Blas, Nayarit (México) por medio de análisis de imágenes Landsat. Ciencias Marina, 32, 523-538.

Blanco, M., Flores-Verdugo, F., Ortiz-Pérez, M., de la Lanza, G., López-Portillo, J., Valdéz-Hernández, I., Agraz-Hernández, C., Czitrom, S., Rivera-Arriaga, E., Orozco, A., Jiménez, G., Benítez, D., Goméz, J. \& González, A. 2011. Diagnóstico funcional de Marismas Nacionales. Universidad Autónoma de Nayarit \& Comisión Nacional Forestal, México, 190 pp.

Blendinger, P. G., Capllonch, P. \& Alvarez, M. E. 2004. Abundance and distribution of raptors in the sierra de San Javier biological park, northwestern Argentina. Ornitologia Neotropical, 15, 501-512.

Bustamante, J. \& Seoane, J. 2004. Predicting the distribution of four species of raptors (Aves: Accipitridae) in southern Spain: statistical models work better than existing maps. Journal of Biogeography, 31, 295-306.

Carmona, R., Hernández-Alvarez, A., Mendoza, L. F. \& Ortega, L. 2011. Observación reciente de Ganso de collar (Branta bernicla nigricans) en Nayarit, México. CICIMAR Oceánies, 26, 65-66.

Carmona, R., Mendoza, L. F., Molina, D., Martínez, P., Vargas, J. \& Cruz, M. 2013. Presencia espacial y temporal de Aramus guarauna (Gruiformes: Aramidae) y de Rostrhamus sociabilis (Accipitriformes: Accipitridae) en la Reserva de la Biosfera Marismas Nacionales, Nayarit, México. Cuadernos de Investigación UNED, $5,129-135$.

Carrete, M., Tella, J. L., Blanco, G. \& Bertellotti, M. 2009. Effects of habitat degradation on the abundance, richness and diversity of raptors across Neotropical biomes. Biological Conservation, 142, 2002-2011.

Cervantes-Abrego, M. 2000. Marismas Nacionales. Pp. 62. In: M. del Coro Arizmendi \& L. Márquez (Eds.). Áreas de importancia para la conservación de aves en México. CONABIO. México, DF., 440 pp.

Clarke, K. R. \& Warwick, R. M. 2001. Change in marine communities: An approach to statistical analysis and interpretation. Primer-E Ltd, U. K, 161 pp.

Collopy, M. W. \& Bildstein, K. L. 1987. Foraging behavior of Northern Harriers wintering in southeastern salt and freshwater marshes. Auk, 104, 11-16.

CONAGUA 2004. Base de datos del Clima en Nayarit. Gerencia Estatal en Nayarit. Departamento de Meteorología.

CONANP 2005. Estudio previo justificativo para el establecimiento del Área Natural Protegida: Área de protección de flora y fauna Marismas Nacionales Nayarit. SEMARNAT. México, DF., 87 pp.

CONANP 2008. Estudio previo justificativo para el establecimiento del Area Natural Protegida: Reserva de la Biosfera Marismas Nacionales Sinaloa. SEMARNAT. México, DF., 62 pp.

Crisci, J. V. \& López, M. F. 1983. Introducción a la Teoría y Práctica de la Taxonomía Numérica. Monografia 26, Serie de Biología, Programa de Monografías Científicas, OEA, Washington D.C. $128 \mathrm{pp}$.

Diario Oficial de la Federación (DOF). 2010a. Norma Oficial Mexi- cana NOM-059-ECOL-2010, Protección ambiental-especies nativas de México de flora y fauna silvestre categorías de riesgos y especificaciones para su inclusión, exclusión o cambio-lista de especies en riesgo. Distrito Federal, México, 78 pp.

Diario Oficial de la Federación (DOF). 2010b. Decreto por el que se declara como área natural protegida, con el carácter de reserva de la biosfera, la región conocida como Marismas Nacionales Nayarit, localizada en los municipios de Acaponeta, Rosamorada, Santiago Ixcuintla, Tecuala y Tuxpan en el Estado de Nayarit. Distrito Federal, México, 43 pp.

Dunn, J. L. \& Alderfer, J. 2011. Field guide to the birds of North America. National Geographic Society. Washington, D. C., 574 pp.

eBrid (aVerAves) 2015. Disponible en: <ebird.org/content/averaves> (consultado en noviembre de 2015).

Escalante, P. 1988. Aves de Nayarit. Coordinación General de Enseñanza Superior. Universidad Autónoma de Nayarit. Nayarit, México.

Escalante, P., Sada, A. M. \& Robles-Gil, J. 2014. Listado de nombres comunes de las Aves de México. Instituto de Biología. UNAM, México, DF., 39 pp.

Fay, F. H., Feder H. M. \& Stocker, F. W. 1977. An estimation on the impact of the pacific walrus population on its food resources in the Bering Sea. Reporte MMC-74/03 of the US Marine Mammals Commission.

Ferguson-Lees, J. \& Christie, D. A. 2001. Raptors of the World. Princeton University Press, Princeton, New Jersey, 320 pp.

Figueroa-Esquivel, E. M. \& Puebla-Olivares, F. 2014. Aves de Sierra de Vallejo, Nayarit, México. Revista Bio Ciencias, 2, 313326.

Fuller, M. R. \& Mosher, J. A. 1987. Raptor survey techniques. Pp. 37-65. In: B. A. Giron, B. A. Millsap, K. W. Cline \& D. M. Bird (Eds.). Raptor management techniques manual. National Wildlife Federation, Washington, DC, USA, $420 \mathrm{pp}$.

Gatto, A., Quintana, F., Yorio, P. \& Lisnizer, N. 2005. Abundancia y diversidad de aves acuáticas en un humedal marino del Golfo San Jorge, Argentina. Hornero, 20, 141-152.

Goodrich, L. J., Crocoll, S. C. \& Senner, S. E. 1996. Broad-winged Hawk (Buteo platypterus). In: A. Poole (Ed.). The Birds of North America. Ithaca: Cornell Lab of Ornithology. Retrieved from the Birds of North America Online: http://bna.birds.cornell.edu/bna/ species/218doi:10.2173/bna.218.

Güemez-Álvarez, C. 2011. Abundancia de las poblaciones de camarones peneidos en las localidades de Antonio Laureles y El Pescadero, del Sistema Lagunar de Agua Brava, Nayarit. Memorias de Residencia Profesional. Instituto Tecnológico de Bahía de Banderas. La Cruz de Huanacaxtle, Nayarit. 42 pp.

Henny, C. J. \& Van Velzen, W. T. 1972. Migration patterns and wintering localities of American Ospreys. Journal of Wildlife Management, 36, 1133-1141.

Hernández-Vázquez, S., Durand-Martínez, B. C., Esparza-Salas, R. \& Valadez-González, C. 2000. Distribución temporal de aves rapaces diurnas en la Reserva "Playón de Mismaloya", Jalisco, México. Revista de Biología Tropical, 48, 1015-1018.

Hernández-Vázquez, S., Rodríguez-Estrella, R., Ramírez-Ortega, F., Loera, J. \& Ortega, M. 2013. Recent increase in the distribution of the snail kite (Rostrhamus sociabilis) along the central 
Pacific Coast of Mexico. Revista Mexicana de Biodiversidad, 84, 388-391.

Howell, S. N. G. 2004. Further observations of birds from Colima and adjacent Jalisco, Mexico. Cotinga, 21, 38-43.

Howell, S. N. G. \& Webb, S. 1995. A Guide to the Birds of Mexico and Northern Central America. Oxford University Press, Oxford, United Kingdom. 837 pp.

Hunt, W. G. 1987. Radio telemetry in the study of raptor habitat selection. Journal of Raptor Research, 21, 144-146.

Iliff, M. J. \& Lovitch, D. .2007. The Changing Seasons: Foods for thought. North American Birds, 61, 208-224.

INEGI 2000. Síntesis de información geográfica del Estado de Nayarit. Instituto Nacional de Estadística Geografía e Informática. México.

Kovacs, J. M., Zhang, C. \& Flores-Verdugo, F. J. 2008. Mapping the condition of mangroves of the Mexican Pacific using C-band ENVISAT ASAR and Landsat optical data. Ciencias Marinas, 34, 407-418.

Krebs, C. J. 1989. Ecological Methodology. Harper \& Row publishers, USA. 645 pp.

López, A. 2012. Deforestación en México: Un análisis preliminar. CIDE. México, DF, 46 pp.

Mañosa, S. \& Pedrocchi, V. 1997. A raptor survey in the Brazilian Atlantic Rainforest. Journal of Raptor Research, 27, 121-122.

Mendoza, L. F., Carmona, R., Arce, N. \& Miramontes, E. 2016. Presencia de grupos invernantes de aguililla de Swainson (Buteo swainsoni) en Nayarit, México. HUITZIL, Revista Mexicana de Ornitología, 17, 120-124.

Ortiz Pulido, R., Gómez de Silva, H., González-García, F. \& Álvarez, A. 1995. Avifauna del Centro de Investigaciones Costeras La Mancha, Veracruz, México. Acta Zoológica Mexicana, 66, 87-118.

Palomera-García, C., Contreras-Martínez, S., Cruz-Rivera, B. Y., Villa Bonilla, B. \& Gómez-Llamas, J. C. 2006. Registros adicionales del Carrao (Aramus guarauna) en el Estado de Jalisco, México. HUITZIL, Revista Mexicana de Ornitología, 7, 23-26.

Pandolfino, E. R., Herzog, M. P., Hooper, S. L. \& Smith, Z. .2011. Winter habitat associations of diurnal raptors in California's central valley. Western Bird, 42, 62-84.

Ramírez-Albores, J. E. 2010. Avifauna de sitios asociados a la selva tropical de la depresión central de Chiapas, México. Acta Zoológica Mexicana, 26, 539-562.

Ramírez-Albores J. E. \& Ramírez Cedillo, M. G. 2002. Avifauna de la región oriente de la Sierra de Huautla, Morelos, México. Anales del Instituto de Biología, Universidad Nacional Autónoma de México, Serie Zoología, 73, 91-111.

RHRAP 2015. Disponible en: <http://www.whsrn.org/es/perfil-de-sitio/marismas-nacionales $>$

Rodríguez-Estrella, R., Donázar, J. A. \& Hiraldo, F. 1998. Raptors as indicators of environmental change in the scrub hábitat of Baja California Sur, Mexico. Conservation Biology, 12, 921-925.
Rodríguez-Estrella, R., Tinajero, R., Partida, A. \& García, R. 2011. Observaciones de una población de Buteo swainsoni que decidió invernar en Baja California Sur. XI Congreso para el Estudio y Conservación de las Aves en México. Mazatlán, Sinaloa. Resumen.

Sánchez-Zapata, J. A. \& Calvo, J. F. 1999. Raptor distribution in relation to landscape composition in semi-arid Mediterranean habitats. Journal of Applied Ecology, 36, 254-262.

Saunders, G. B. \& Saunders, Ch. D. 1981. Waterfowl and their Wintering Grounds in México, 1937-64. Fish and Wildlife Service. U. S. Department of the Interior. Resource Publication, 138, $1-151$.

SEMARNAT 2007. ¿Y el medio ambiente? Problemas en México y el mundo. SEMARNAT. México. 192 pp.

Siegel, S. \& Castellan, Jr., N. J. 1988. Nonparametric statistics for the behavioral sciences. McGraw-Hill, Singapur. 399 pp.

Smallwood, J. A. \& Bird, D. M. 2002. American Kestrel (Falco sparverius). In: A. Poole (Ed.). The Birds of North America. Ithaca: Cornell Lab of Ornithology; Retrieved from the Birds of North America Online: http://bna.birds.cornell.edu/bna/species/602.

Sykes, Jr., P. W., Rodgers, Jr., J. A. \& Bennetts, R. E. 1995. Snail Kite (Rostrhamus sociabilis). In: A. Poole (Ed.). The Birds of North America, A. Poole (ed). Ithaca: Cornell Lab of Ornithology; Retrieved from the Birds of North America Online: http://bna.birds. cornell.edu/bna/species/171.

Thiollay, J. M. 1989. Area requirements for the conservation of rain forest raptors and game birds in French Guiana. Conservation Bio$\log y 31,128-137$.

van Perlo, B. 2006. Birds of Mexico and Central America. Princeton University Press, Princeton, New Jersey. 336 pp.

Vannini, J. P. 1989. Neotropical raptors and deforestation: notes on diurnal raptors at Finca El Faro, Quetzaltenango, Guatemala. Journal of Raptor Research, 23, 27-38.

Vázquez, L., Moya, H. \& del Coro Arizmendi, M. 2009. Avifauna de la selva baja caducifolia en la cañada del río Sabino, Oaxaca, México. Revista Mexicana de biodiversidad, 80, 535-549.

Vázquez-Pérez, J. R., Enríquez, P. L. \& Rangel-Salazar, J. L. 2009. Diversidad de rapaces diurnas en la Reserva de la Biosfera Selva del Ocote, Chiapas, México. Revista Mexicana de Biodiversidad, 80, 203-209.

Vergara, P. 2010. Time-of-day bias in diurnal raptor abundance and richness estimated by road surveys. Revista Catalana d'Ornitologia, 26, 22-30.

Williams, C. K., Applegate, R. D., Lutz, R. S. \& Rusch, D. H. 2000. A comparison of raptor densities and habitat use in Kansas cropland and rangeland ecosystems. Journal of Raptor Research, 34, 203-209.

Zurita, G. A. \& Bellocq, M. I. 2007. Pérdida y fragmentación de la selva Paranaense: efectos sobre las aves rapaces diurnas. Hornero, 22, 141-147. 